\title{
Nanotube confinement denatures protein helices Supporting Information
}

\author{
Eric J. Sorin ${ }^{1} \&$ Vijay S. Pande ${ }^{1,2^{*}}$ \\ Departments of ${ }^{1}$ Chemistry and ${ }^{2}$ Structural Biology \\ Stanford University, Stanford, CA 94305-5080 \\ March 2006
}

\begin{abstract}
*Corresponding Author:
Vijay S. Pande

Assistant Professor, Department of Chemistry,

Structural Biology Department and SSRL85

Stanford University, Stanford, CA, 94305-3080

Phone: 650-723-3660

Fax: 650-725-0259

URL: http://pande.stanford.edu

Email: pande@stanford.edu
\end{abstract}

\section{Methods}

\section{(a) Molecular dynamics simulations}

We have recently shown that simulating absolute equilibrium at the ensemble level is now tractable for helical peptide systems, including an explicit representation of the solvent, using the highly optimized GROMACS molecular dynamics suite ${ }^{1}$ modified for the our Folding@ $@$ Home $^{2}$ distributed computing network. ${ }^{3,4}$ Our previous results showed that the AMBER-99 $\phi$ all-atom force field, in conjunction with the TIP3P water model, ${ }^{5}$ performed well at reproducing the experimentally identified kinetic and thermodynamic character of the capped 23-residue polyalanine based $\mathrm{F}_{\mathrm{s}}$ peptide $\left(\mathrm{Ace}_{-} \mathrm{A}_{5}\left[\mathrm{AAAR}^{+} \mathrm{A}\right]_{3} \mathrm{~A}\right.$ $\mathrm{NMe}$ ). The capped $\mathrm{A}_{21}$ homopolymer (Ace- $\mathrm{A}_{21}-\mathrm{NMe}$ ), which was shown to mimic the behavior of the arginine-substituted $\mathrm{F}_{\mathrm{s}}$ in silico, was simulated inside of fully solvated carbon nanotubes (CNTs) with increasing diameter, thereby allowing us to avoid potential artifacts due to confined counterions and look solely at the phenomenon of confinement-induced changes in peptide conformational equilibria. This peptide also maintains simplicity by removing potential effects due to the chemical or physical nature of ARG sidechains and allowing for a smaller minimum CNT diameter.

Six CNT models were constructed and parameterized using the most relevant $\mathrm{sp}^{2}$ atom type from the AMBER force field (CA). We note that all $\mathrm{sp}^{2}$ atom types in a given force field generally have identical Lennard-Jones parameters, and this is true of the AMBER potential employed in this study. The six models were of chirality $(m, n=m)$, where $\mathrm{m}=\{11,13,15,19,22,26\}$, yielding approximate tube diameters of 14.9, 17.6, 20.3, 25.8, 29.8, and 35.3 A respectively. While the initial length of each CNT was 89.3 $\AA$, the nanotube structures were held fixed and aligned using periodic boundary conditions to produce infinite nanotube constructs, thus avoiding edge effects and artifacts that may result from pressure scaling. Fully extended $\left(\phi=180^{\circ}, \psi=180^{\circ}\right)$ and fully $\alpha$-helical $\left(\phi=-57^{\circ}, \psi=-47^{\circ}\right)$ peptide conformations were then centered in each CNT model and solvated with TIP3P water, ${ }^{5}$ followed by energy minimization using a steepest descent algorithm and solvent annealing for $500 \mathrm{ps}$ with peptide atoms constrained. A 
second solvation and annealing step was used to ensure full solvation of the infinite CNTs (see "Nanotube solvation" below). For all CNTs, the periodic box vectors perpendicular to the CNT axis were $10 \AA$ larger than the diameter of the CNT, thereby accounting for long range interactions that may be affected by external solvent. We performed all simulations in the NVT-isothermal ensemble at $305 \mathrm{~K}$ (the approximate midpoint temperature detected by circular dichroism ${ }^{6}$ and ultraviolet resonance $\operatorname{Raman}^{7}$ ), with frames stored every 100 ps.

\section{(b) Nanotube solvation}

An important aspect of this work is the modeling of solvated nanotubes. As stated above, all CNT models were aligned to generate infinite nanotubes within the periodic boundary construct, thereby removing potential end-effects from the simulations. However, this method also results in closed CNT systems with no transport of water into or out of the CNTs. Accounting for internal water density is thus an important factor that could result in simulation artifacts, and we therefore conducted several CNT test simulations without the peptide inserted.

We first simulated the $(15,15)$ nanotube in non-infinite tube conditions inside a periodic box of TIP3P water with sides $30 \AA \times 30 \AA \times 101 \AA$. As expected, water density was highest near the center of the tube, tapering off near the edges. We then ran this same system using infinite CNT alignment and a single solvation-annealing step. As expected, ${ }^{8-11}$ this resulted in initial bubble formation near the tube ends, with the bubble appearing mobile throughout the simulation. To best generate model peptide confinement in a generalizable way and to remove potential simulation artifacts, this bubble was removed by performing a second solvation-annealing step. In this simulation, a uniform internal water density was observed, with only a modest density increase $(<$ $10 \%$ ) compared to the simulation using a single solvation step (which included bubble formation). For the peptide-CNT simulations reported in our Letter, the excluded volume of the inserted peptide also counteracts this density increase upon solvation, and we thus feel that our method of double solvation provides the most reasonable and generalizable model of peptide-solvent confinement.

As described in our Letter, the formation of the $S_{1}$ and $S_{2}$ solvation layers was also a somewhat expected phenomenon based on previous CNT studies. ${ }^{8-11}$ As with the mean bulk helicity, the presence and location of these hydration layers will depend on the water model and simulation methodology employed. Furthermore, while the effective diameter of the smallest CNT is less than that of the ideal helix, two factors must be considered. First, that the ideal helix is not readily observed in our simulations - as we describe here and in our previous articles on the helix-coil transition, the helix is a very rapidly fluctuating conformation in which the mean helical segment length is only approximately 5 residues. In this small CNT we observe a mean helical content (across the entire peptide) that is very near this value. Only small helical nuclei are present, allowing the remaining chain to fluctuate in more extended states. Second, the solvation shells described in our article, and indeed in other studies of CNT solvation, are not physically impermeable barriers, and therefore can be interrupted by the peptide. This crossing of hydration shells is simply disfavored (free) energetically.

\section{(c) Ensemble equilibrium and simulation analysis}

Secondary structure was determined using the Dictionary of Secondary Structure for Proteins (DSSP). ${ }^{12}$ While there are many ways to define helicity, DSSP does so by considering intra-peptide hydrogen bonding. This algorithm is thus the most logical 
application to use in comparing simulated helicity to the simple model of hydrogen bonding put forth in our letter. DSSP also distinguishes between $\alpha$-helix and 310 -helix types, and the majority of helical content observed in our simulations are of the latter type. While further simulations to investigate this effect are ongoing, we believe it to result from the inherent internal pressure of the solvated CNT system and note that most experimental methods used to investigate secondary structure cannot generally distinguish between these two conformational types.

Hydrogen bond (HB) counts were determined using the g_hbond module of the GROMACS package ${ }^{1}$ with the default donor-acceptor radius of $2.5 \AA$ and donorhydrogen-acceptor angle of $60^{\circ}$. Errors in ensemble averaged hydrogen bonding values were taken as the maximum deviations from the ensemble mean after splitting the data into 10 independent subsets, and all hydrogen bonding probability distributions were verified as being unimodal and narrowly peaked.

As in our previous reports on simulations of the helix-coil transition, we examined the total mean helical content, the mean contiguous helical content, and the mean number of helical segments in each of our simulated ensembles to determine convergence to ensemble equilibrium. We find that confining the helical peptide greatly slows the convergence process, and have therefore collected far greater sampling of confined helical peptides than was done for our previous works. While this communication does not report on the kinetics of this system (manuscript in progress), the slow nature of this convergence impacts the quantity and accuracy of equilibrium data available for analysis. In each system studied, we found that the ensemble starting in the ideal helical conformation relaxed to equilibrium on the $50 \mathrm{~ns}$ timescale, while the ensemble starting from the extended state required $>250 \mathrm{~ns}$, leaving no suitable quantity of equilibrium data from the extended ensemble. In all cases, the extended ensemble approaches the equilibrium values predicted by the ensemble starting in the ideal helical conformation. We therefore based equilibrium calculations reported herein solely on the equilibrated ensembles starting in the ideal helical conformation and including only data taken well after ensemble equilibrium was reached.

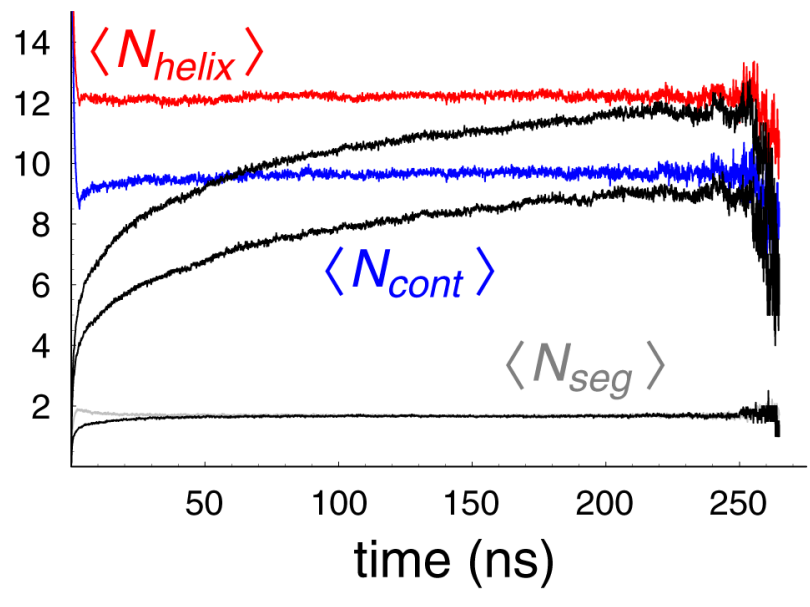

Figure S1. Example time-series of ensemble averaged helical properties used to monitor convergence (shown for the $(15,15)$ nanotube system with $\mathrm{d}=20.3 \AA$ ), including the mean total helicity (red), mean contiguous helicity (blue), and mean number of helical segments (gray). Colored and black time-series correspond to ensembles started in the helical and extended conformations respectively. Noise at long times is due to a limited number of trajectories reaching those times. While ensembles started in the extended state do not reach absolute ensemble equilibrium, those started in the helical state equilibrate more rapidly and are used to calculate equilibrium properties.

\section{(d) Calculation of relative solvent entropy}

To directly measure the solvent entropy in our simulations, we employ the Nadler \& Krausche "square-water" independent bond (IB) model of aqueous hydrogen bonding. ${ }^{13}$ This statistical mechanical model includes the partition function 


$$
\mathrm{Z}_{I B}=\left(1+e^{\beta \varepsilon}\right)^{2 N_{H_{2} O}},
$$

where $\beta=1 / \mathrm{kT}, \mathrm{k}$ is Boltzmann's constant, $\mathrm{T}$ is absolute temperature, $N_{\mathrm{H}_{2} \mathrm{O}}$ is the total number of water molecules inside the tube, and $\varepsilon$ is the change in energy upon hydrogen bonding formation. From the IB model, the mean number of solvent-solvent hydrogen bonds per water molecule is given by

$$
\frac{\left\langle \# \mathrm{HB}_{w w}\right\rangle}{N_{\mathrm{H}_{2} \mathrm{O}}}=\frac{1}{\varepsilon \cdot N_{\mathrm{H}_{2} \mathrm{O}}} \frac{d}{d \beta} \ln \mathrm{Z}_{I B} .
$$

For the canonical ensemble (N,V,T held constant, as done in our simulations), entropy is defined as

$$
S_{\mathrm{H}_{2} \mathrm{O}}=\mathrm{k} \ln \mathrm{Z}_{\mathrm{IB}}+\frac{\mathrm{U}}{\mathrm{T}}=\mathrm{k} \ln \mathrm{Z}_{\mathrm{IB}}-\frac{1}{\mathrm{~T}} \frac{d}{d \beta} \ln \mathrm{Z}_{I B} .
$$

We substitute equations (1) and (2) above to yield the solvent entropy as

$$
\frac{\mathrm{T} \cdot S_{\mathrm{H}_{2} \mathrm{O}}}{N_{\mathrm{H}_{2} \mathrm{O}}}=2 \mathrm{RT} \ln \left(1+\mathrm{e}^{\beta \varepsilon}\right)-\varepsilon \frac{\left\langle \# \mathrm{HB}_{\mathrm{ww}}\right\rangle}{N_{\mathrm{H}_{2} \mathrm{O}}},
$$

and calculate the change in solvent entropy as compared to bulk water outside the CNTs. Here $\mathrm{R}=\mathrm{k} \cdot \mathrm{N}_{\mathrm{a}}$ is the universal gas constant and, for simplicity, we assume $\varepsilon=-6.0$ $\mathrm{kcal} / \mathrm{mol}$, as discussed recently by Baldwin. ${ }^{14}$ Similar (negative) values of $\varepsilon$ do not change the qualitative diameter dependence of the solvent entropy. Taking the number of water molecules and the mean hydrogen bond counts from our simulations yields the entropy of only the solvent based specifically on each nanotube system.

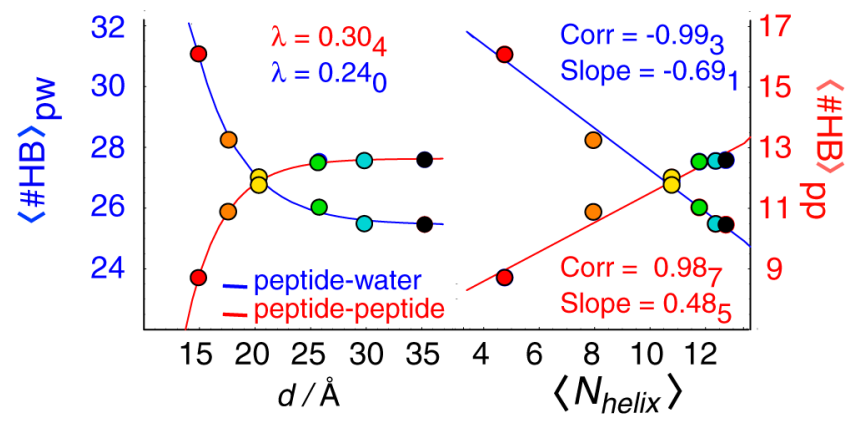

(e) Two-state model for helix hydrogen bonds

The two-state model for peptide-peptide hydrogen bonding follows the two-state IB model described above and assumes the partition function

$$
\mathrm{Z}_{\mathrm{HB}}=\left(1+e^{\beta \cdot \Delta \mathrm{G}(d)}\right)^{\left\langle N_{\text {helix }}(b u l k)\right\rangle},
$$

where $\beta=1 / \mathrm{kT}, \mathrm{k}$ is Boltzmann's constant, $\mathrm{T}$ is absolute temperature, $\Delta \mathrm{G}(d)$ is the free energy change associated with forming a peptide-peptide HB (which depends on the 
diameter of the tube), and $\left\langle N_{\text {helix }}(\right.$ bulk $\left.)\right\rangle$ is the mean number of peptide-peptide HBs expected in bulk solvent simulations (as $\mathrm{d} \rightarrow \infty$ ). As follows from statistical mechanics, we can take derivatives of the partition function to obtain the mean number of helical HBs in the form

$$
\left\langle\mathrm{N}_{\text {helix }}\right\rangle=\frac{1}{\Delta \mathrm{G}(d)} \frac{d}{d \beta} \ln \mathrm{Z}_{\mathrm{HB}}=\frac{\left\langle N_{\text {helix }}(\text { bulk })\right\rangle}{1+e^{-\beta \cdot \Delta \mathrm{G}(d)}},
$$

where

$$
\Delta \mathrm{G}(d)=\varepsilon_{\mathrm{HB}}+\mathrm{kT} \ln \left[\pi\left(d_{\mathrm{eff}} / 2\right)^{2} / A_{\mathrm{H}_{2} \mathrm{O}}\right]
$$

as specified in our Letter. In this equation, $\pi\left(d_{\mathrm{eff}} / 2\right)^{2}$ is the effective cross sectional area of the infinite CNT. We thus obtain a prediction of $\left\langle N_{\text {helix }}(d)\right\rangle$ with no free parameters to fit.

\section{Additional Discussion}

\section{Limitations of model}

While we employ atomistic detail and extensive sampling in modeling nanotube confinement of helical peptides, our model of the confining CNT structure remains relatively simple. The goal of this work is to investigate the physical chemistry of confined peptide conformational equilibria. However, additional molecular-level details may result in further refinement in our understanding. These include the use of sequences with polar or charged sidechains (with explicit counterions) and those with varying hydrophobic character, as well as the incorporation of a non-constrained CNT model and other more heterogeneous confining vessels.

Although water models differ, the behavior observed herein is expected to be essentially independent of the model, with minor differences in the parameterization of molecular water resulting in minor differences in the calculated values described in our Letter. Finally, the agreement between our simple theory and detailed simulations suggest that details such the nature of the water model may not be significant.

\section{References}

1. Lindahl, E., Hess, B. \& van der Spoel, D. GROMACS 3.0: a package for molecular simulation and trajectory analysis. Journal Of Molecular Modeling 7, 306-317 (2001).

2. Zagrovic, B., Sorin, E. J. \& Pande, V. $\beta$-hairpin folding simulations in atomistic detail using an implicit solvent model. Journal Of Molecular Biology 313, 151169 (2001).

3. Sorin, E. J. \& Pande, V. S. Exploring the Helix-coil Transition via All-atom Equilibrium Ensemble Simulations. Biophysical Journal 88, 2472-2493 (2005).

4. Sorin, E. J. \& Pande, V. S. Empirical Force Field Assessment: the Interplay Between Backbone Torsions and Non-covalent Term Scaling. Journal of Computational Chemistry 26, 682-690 (2005). 
5. Jorgensen, W. L., Chandrasekhar, J., Madura, J. D., Impey, R. W. \& Klein, M. L. Comparison of simple potential functions for simulating liquid water. Journal of Chemical Physics 79, 926-935 (1983).

6. Thompson, P. A., Eaton, W. A. \& Hofrichter, J. Laser Temperature Jump Study of the Helix-Coil Kinetics of an Alanine Peptide Interpreted with a 'Kinetic Zipper' Model. Biochemistry 36, 9200-9210 (1997).

7. Ianoul, A., Mikhonin, A., Lednev, I. K. \& Asher, S. A. UV Resonance Raman Study of the Spatial Dependence of $\alpha$-Helix Unfolding. Journal of Physical Chemistry A 106, 3621-3624 (2002).

8. Gordillo, M. C. \& Marti, J. Hydrogen bond structure of liquid water confined in nanotubes. Chemical Physics Letters 329, 341-345 (2000).

9. Hummer, G., Rasaiah, J. C. \& Noworyta, J. P. Water conduction through the hydrophobic channel of a carbon nanotube. Nature 414, 188-190 (2001).

10. Sen, S. Exploring Structure and Energetics of a Helix-Forming Oligomer by Molecular Modeling and Molecular Dynamics Simulation Methods: Dynamics of Water in a Hydrophobic Nanotube. Journal of physical Chemistry B 106, 1134311350 (2002).

11. Kolesnikov, A. I. et al. Anomalously Soft Dynamics ofWater in a Nanotube: A Revelation of Nanoscale Confinement. Physical Review Letters 93, 035503/1-4 (2004).

12. Kabsch, W. \& Sander, C. Dictionary of protein secondary structure: pattern recognition of hydrogen-bonded and geometrical features. Biopolymers 22, 25772637 (1983).

13. Nadler, W. \& Krausche, T. Universality in hydrogen-bond networks. Physical Review A 44, R7888-R7890 (1991).

14. Baldwin, R. L. In Search of the Energetic Role of Peptide Hydrogen Bonds. Journal Of Biological Chemistry 278, 17581-17588 (2003). 